\title{
A decision support system based on radiomics and machine learning to predict the risk of malignancy of ovarian masses from transvaginal ultrasonography and serum CA-125
}

Valentina Chiappa ${ }^{1 \dagger}$, Matteo Interlenghi ${ }^{2+}$, Giorgio Bogani ${ }^{1}$, Christian Salvatore ${ }^{2^{*}}$ (D), Francesca Bertolina ${ }^{1}$, Giuseppe Sarpietro ${ }^{1}$, Mauro Signorelli ${ }^{1}$, Dominique Ronzulli ${ }^{3}$, Isabella Castiglioni ${ }^{4}$ and Francesco Raspagliesi ${ }^{1}$

\begin{abstract}
Background: To evaluate the performance of a decision support system (DSS) based on radiomics and machine learning in predicting the risk of malignancy of ovarian masses (OMs) from transvaginal ultrasonography (TUS) and serum CA-125.

Methods: A total of 274 consecutive patients who underwent TUS (by different examiners and with different ultrasound machines) and surgery, with suspicious OMs and known CA-125 serum level were used to train and test a DSS. The DSS was used to predict the risk of malignancy of these masses (very low versus medium-high risk), based on the US appearance (solid, liquid, or mixed) and radiomic features (morphometry and regional texture features) within the masses, on the shadow presence (yes/no), and on the level of serum CA-125. Reproducibility of results among the examiners, and performance accuracy, sensitivity, specificity, and area under the curve were tested in a real-world clinical setting.

Results: The DSS showed a mean 88\% accuracy, 99\% sensitivity, and 77\% specificity for the 239 patients used for training, cross-validation, and testing, and a mean 91\% accuracy, 100\% sensitivity, and 80\% specificity for the 35 patients used for independent testing.
\end{abstract}

Conclusions: This DSS is a promising tool in women diagnosed with OMs at TUS, allowing to predict the individual risk of malignancy, supporting clinical decision making.

Keywords: Artificial intelligence, Machine learning, CA-125 antigen, Ovarian neoplasms, Ultrasonography

\footnotetext{
* Correspondence: salvatore@deeptracetech.com

t alentina Chiappa and Matteo Interlenghi contributed equally to this work

${ }^{2}$ DeepTrace Technologies S.R.L., Milan, Italy

Full list of author information is available at the end of the article
}

\section{Springer Open}

(c) The Author(s). 2021 Open Access This article is licensed under a Creative Commons Attribution 4.0 International License, which permits use, sharing, adaptation, distribution and reproduction in any medium or format, as long as you give appropriate credit to the original author(s) and the source, provide a link to the Creative Commons licence, and indicate if changes were made. The images or other third party material in this article are included in the article's Creative Commons licence, unless indicated otherwise in a credit line to the material. If material is not included in the article's Creative Commons licence and your intended use is not permitted by statutory regulation or exceeds the permitted use, you will need to obtain permission directly from the copyright holder. To view a copy of this licence, visit http://creativecommons.org/licenses/by/4.0/. 


\section{Key points}

- A decision support system (DSS) was developed for predicting malignancy-risk level of ovarian masses.

- The DSS is based on transvaginal ultrasound imaging and serum CA-125 level.

- Radiomics and machine learning techniques were implemented.

- The DSS showed $91 \%$ accuracy, $100 \%$ sensitivity, and $80 \%$ specificity in independent testing.

- The DSS allows predicting individual risk level of ovarian mass malignancy.

\section{Background}

Ovarian cancer $(\mathrm{OC})$ is one of the most lethal cancers in women [1], with over 295,400 cases diagnosed worldwide in 2018 and 184,800 deaths [2]. The lack of accurate screening and diagnostic tools, the scarce symptomatology, abnormal cell metabolism [3], and the rapid spread of disease are the main causes of lethality. Biological markers, such as cancer antigen-125 (CA-125), have been shown highly sensitive but poorly specific for screening, and qualitative transvaginal ultrasonography (TUS) are not informative enough to detect $\mathrm{OC}$ at the early stage in the general population $[4,5]$. To date, no screening test has proven to reduce ovarian cancer mortality, and ovarian cancer screening is not recommended by any scientific society in the general population. Some societies recommend screening with CA-125 and TUS in highrisk women (e.g., carriers of BRCA1 and other genes mutations) [6].

The high false positive rate of diagnostic methods leads to often useless surgeries for benign masses at final histology and to the lack of centralisation of oncological cases with worsening of the patient's prognosis [7]. An adequate and reproducible preoperative diagnosis is therefore of paramount importance [8].

The adnexal ovarian masses appear to have particular characteristics at TUS, helpful in the diagnosis to expert operators. For example, masses with predominantly solid composition, irregular in shape, large, and not presenting underlying shadows are more probably malignant. Based on these characteristics, guidelines support specialised clinicians to classify the risk of cancer of ovarian masses on the bases of only ultrasound features as is for the International Federation of Gynecology and Obstetrics (FIGO) classification [9] or with a combination of ultrasound and biological markers, as is for the "risk of malignancy index" (RMI) [10] or "assessment of different neoplasias in the adnexa" (ADNEX) [11].

These classifications are helpful in high and low risk groups but most of them require the knowledge of a specific terminology [12] and a certain level of experience of the examiner in the assignment of echogenic and structural features to OMs, and are inconclusive for lesions assigned with an intermediate risk.

Radiomics is a quite recent but popular image processing technique applied to radiological medical images, including those obtained with TUS [13-15], based on the extraction of quantitative data about lesion morphology and texture features. These features have been shown to be correlated to the pathophysiology of cancer tissue in a large number of oncological diseases [16] and used in multivariate classification models to discriminate cancerous from non-cancerous tissues [17].

In the case of $\mathrm{OC}$, we have recently developed a classification model based on radiomics applied to ultrasonography to automatically classify lesions with proven benign versus malignant histopathology on surgical specimens, allowing a stratification of low versus high risk of cancer with a mean $84 \%$ accuracy, $79 \%$ sensitivity, and $86 \%$ specificity [18].

In this work, we evaluated the performance of such classification model when used in combination with CA125 in predicting the risk level of malignancy of OMs (very low risk, medium-high risk), to be used by specialised clinicians as decision support system (DSS) for a personalised diagnostic and treatment pathway for individual patients. We then tested generalisation and reproducibility of such DSS on a patients' cohort independent from that used for the development of the previous system, being the DSS used by different examiners with different experience.

\section{Methods}

\section{Study design and study population}

This is a single-centre, observational, retrospective, and prospective clinical study. The study population includes two cohorts.

The first cohort is retrospective and inclusion criteria were as follows: consecutive women with age $\geq 18$ years who underwent TUS and were diagnosed with OMs, then scheduled for surgery within 2 weeks after TUS examination at Fondazione IRCCS Istituto Nazionale dei Tumori di Milano from January 1, 2017, to December 31, 2019. CA-125 serum level was required.

The second cohort was prospective and inclusion criteria were as follows: consecutive women with age $\geq 18$ years who underwent TUS and were diagnosed with OMs, then scheduled for CA-125 level test and surgery within 2 weeks after US examination at Fondazione IRCCS Istituto Nazionale dei Tumori di Milano from January 1, 2021, to April 31, 2021.

Patients in the prospective cohort and patients still alive and traceable in the retrospective cohort signed the study-specific informed consent. For deceased or untraceable patients, the Principal Investigator has filled in the "replacement form for informed consent" approved 
by the ethics committee, in compliance with DGPR 679/ 2016. The study protocol was approved by the Ethical Committee (Protocol MULTIAROMA INT. N157/20 Scientific Responsible Dott. Valentina Chiappa, approved 22/07/2020).

A gynaecologist with more than 10 years of experience (senior experience) performed all US examinations and diagnosis at TUS, saving the $2 \mathrm{D}$ image frames in DICOM format with the patient's ovarian mass acquired in longitudinal and perpendicular sections, respectively. Ultrasound competence was not assessed using quantitative scale considering pass/fail scores (e.g., assessment of ultrasound skills).

For both the cohorts, final histology after surgery was considered as standard reference for the definite diagnosis.

\section{The DSS}

The DSS was based on an ensemble of three radiomic machine learning models, specifically designed to classify the risk level for (i) fully solid OMs ("solid masses"), (ii) fully liquid OMs ("cystic masses"), and (iii) OMs with solid and liquid components ("mixed masses"). Such radiomic machine learning models have been previously proposed and tested on the first patient cohort in [18]. Two hundred and sixty-nine (269) stable radiomics features have been found as stable predictors to characterise the echogenic structure and morphometry of solid OMs by the first model, 278 for the liquid masses by the second model, and 306 for the third one customised for the lesions with mixed solid and liquid components.

An improvement in the design of such radiomic model is considered in this work by integrating the malignancy risk predicted by each of the three TUS radiomic models [18] with the information on the presence/absence of acoustic shadows, and combining the malignancy risk predicted by such TUS-based model with the serum CA-125 level, considering two different thresholds depending from the menopausal status (premenopause/ postmenopause as correctors for CA-125 level). Indeed, the presence of acoustic shadows [12] among solid components has been well acknowledged associated with an OM classification of very low risk of malignancy [11]. Thus, we integrated the malignancy risk predicted by the model trained on radiomic features within the

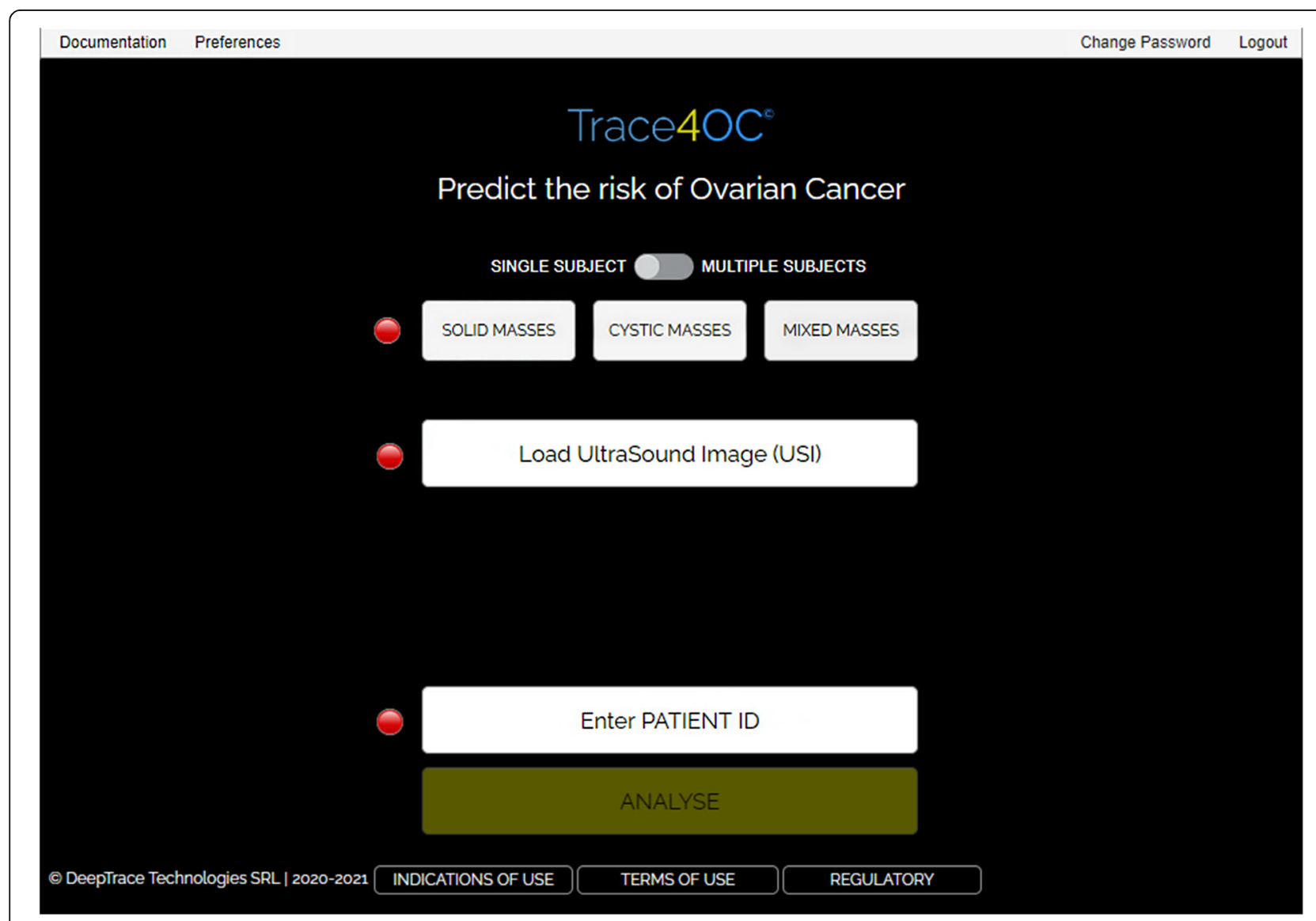

Fig. 1 Software interface for the mass types and transvaginal ultrasound image selection 
ovarian mass with the information on the presence/absence of acoustic shadows near the mass that does not modify such risk (if acoustic shadows are absent) or reduce such risk (if acoustic shadows are present). On the other hand, for patients diagnosed with OMs at TUS, serum CA-125 level higher than of $71 \mathrm{U} / \mathrm{mL}$ (double compared to the upper normal level) for postmenopausal women [19] and $200 \mathrm{U} / \mathrm{mL}$ for premenopausal women [20, 21], respectively, have been found associated to a classification of medium-high risk of malignancy.

Specifically, according to such a combined risk approach, in this work, we defined the following DSS and predicted risk:

- When an OM is shown by TUS without acoustic shadows and the serum CA-125 level is below the threshold, the DSS predicts the risk level of malignancy based on the classification of the radiomic models (very low risk or medium-high risk);
- When an OM is shown by TUS with acoustic shadows and the serum CA-125 level is below the threshold, the DSS predicts a very low risk of malignancy;

- When an OM is shown by TUS with or without acoustic shadows and the serum CA-125 level is above the threshold, the DSS predicts a mediumhigh risk of malignancy.

The DSS was implemented in a stand-alone software tool that can be installed on dedicated Microsoft windows workstations, in an on-premise configuration, with the following minimum characteristics: operative system windows 10 , processor Intel i5 $\times 8664$, RAM of $8 \mathrm{~GB}$, and $8 \mathrm{~GB}$ of free space on the hard drive.

For each patient's ovarian mass, the number of TUS images to upload in the software is only one: the 2D image frame saved in DICOM format with the mass acquired in the longitudinal section.



Fig. 2 Manual segmentation interface 


\section{Presence of Acoustic Shadows: YES $\checkmark$ \\ Post-menopausal: YES \\ Serum CA-125 level (U/mL): [Not mandatory] \\ OK \\ Cancel}

Fig. 3 Interface for supplementary information: presence of acoustic shadows, subject menopausal state, and serum CA-125 level

The software tool allows high-resolution viewing of the uploaded TUS image, visual inspection of liquid and solid components of the ovarian mass as well as the presence or absence of acoustic shadow, and fast segmentation of the OMs, through an easy-to-use graphical interface (Fig. 1 and Fig. 2). The segmentation modality is manual, and a process of random manipulations of the contour (small geometrical modifications of the manual contour creating different contours from different operators) is automatically performed by the software to minimise the dependency of the radiomic analysis from the manual segmentation of different operators [18]. Robust radiomic features are automatically calculated and selected by the software for the manipulated segmented mass on the TUS image according to the specific mass type as described in our previous work [18] (269 features for solid masses, 278 features for cystic masses, and 306 features for mixed masses). Such radiomic features are used by one of the three machine learning models, according to the mass type, to predict the risk of malignancy of the mass based only on the TUS radiomic features within the mass. Finally, the supplementary information (i.e., the presence/absence of acoustic shadows at ultrasonography, the menopausal status of the woman, and the serum CA-125 level) is provided to the software (Fig. 3) that predicts the risk of malignancy of the mass (Fig. 4).

In this work, the diagnostic performance and reproducibility of the DSS software was tested by two TUS examiners with different levels of experience: the first one (Examiner 1) was a gynaecologist with less than 2 years of experience (intermediate experience), the second one (Examiner 2) was the gynaecologist with more than 10 years of experience (senior experience).

\section{Statistical analysis}

Data are presented as median and interquartile range (IQR), for patients' age, frequencies and percentage for mass characteristics, and premenopausal or menopausal status. Diagnostic performances were obtained in terms of sensitivity, specificity, and accuracy comparing the results of the DSS (very low versus medium-high) versus histopathology reference standard (benign versus

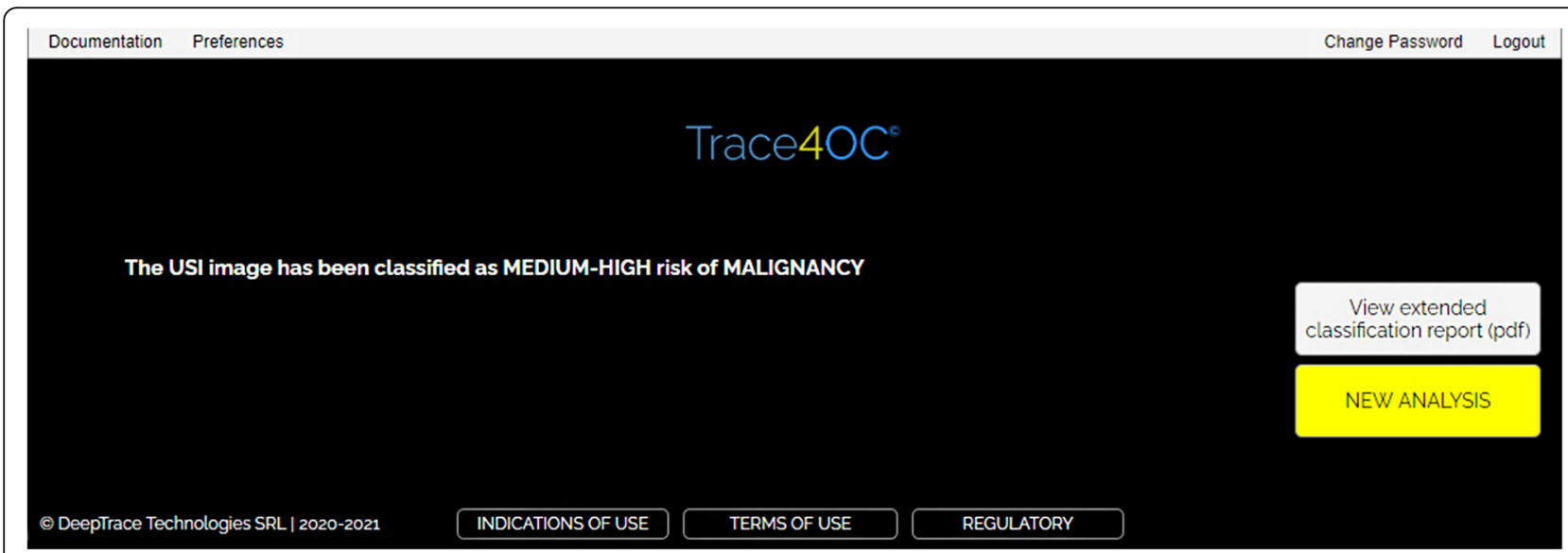

Fig. 4 Interface of the classification results 
Table 1 First cohort population

\begin{tabular}{lllllll}
\hline & $\begin{array}{l}\text { Number of } \\
\text { patients }\end{array}$ & $\begin{array}{l}\text { Mass } \\
\text { type }\end{array}$ & $\begin{array}{l}\text { Median age } \\
\text { (IQR) }\end{array}$ & $\begin{array}{l}\text { Postmenopausal } \\
\text { (\%) }\end{array}$ & $\begin{array}{l}\text { Malignant } \\
\text { (\%) }\end{array}$ & $\begin{array}{l}\text { Malignant, median age } \\
\text { (IQR) }\end{array}$ \\
\hline First cohort (239 & 93 & Solid & $56(21)$ & $58(62.4 \%)$ & $57(61.3 \%)$ & $51(20)$ \\
women) & 67 & Liquid & $51(22)$ & $36(53.7 \%)$ & $12(17.9 \%)$ & $54(19)$ \\
& 79 & Mixed & $58(23)$ & $54(68.4 \%)$ & $54(68.4 \%)$ & $58(21)$ \\
\hline
\end{tabular}

IQR Interquartile range

malignant), with $95 \%$ confidence intervals (CI), calculated according to the binomial distribution.

\section{Results}

\section{Population}

From the first cohort, we retrospectively tested 239 women with available TUS images of OMs, serum CA125 levels, and histopathological results after surgery. TUS images were obtained from a VOLUSON-E8 system (General Electric Healthcare, Chicago, USA). Median age of the patients was 55 years with an IQR of 22 (minimum 18, maximum 84).

Most of patients (61\%) referred symptoms before TUS: pelvic or abdominal pain (50\%), bloating (20\%), increase in abdominal circumference (19\%), weight gain or loss $(18 \%)$, weakness nausea (17\%), irregular menstrual periods $(14 \%)(13 \%)$, abnormal uterine bleeding $(6 \%)$, dyspareunia (3\%). The characteristics of the three groups of OMs (solid, liquid and mixed) for this first cohort are summarised in Table 1 . The majority of patients (62\%) were postmenopausal.

From the second, independent cohort, we prospectively tested 35 women with available TUS images of OMs, serum CA-125 levels, and histopathological results treated from January 1, 2021, to April 30 2021. TUS images were obtained from a HERA W10 system (Samsung, Seoul, South Korea). Median age of the patients was 50 years with an IQR of 25 (minimum 18, maximum 73). The characteristics of the three groups of OMs (solid, liquid, and mixed) for this second cohort are summarised in Table 2; $57 \%$ of patients were postmenopausal.

Histological characteristics of the OMs after surgery and International Federation of Gynecology and Obstetrics (FIGO) stage in case of malignancy for both cohorts 1 and 2 are summarised in Table 3.

\section{DSS performance}

The performance metrics of DSS (specificity, sensitivity, accuracy, positive predictive value, negative predictive value), with $95 \% \mathrm{CI}$ in square brackets, are shown in Table 4 for the first and second cohorts as well as for the two different examiners using DSS on the same images of the same patients.

Serum CA-125 levels above thresholds were found for 81 women (71 and 10 from the first and second cohorts, respectively), corresponding to $30 \%$ of the 274 OMs. Acoustic shadows were identified in 46 TUS (37 and 9 from the first and second cohorts, respectively), corresponding to $17 \%$ of the 274 OMs. Serum CA-125 levels below threshold was found in 36 of the 46 women with acoustic shadows in their TUS (30 and 6 from the first and second cohorts, respectively), corresponding to $13 \%$ of the 274 OMs. The classification of the remaining 157 OMs (138 and 19 from the first and second cohorts, respectively, corresponding to $57 \%$ of the 274 OMs) depended on the radiomic models.

Sample images from different settings are shown in Figs. 5, 6, 7, 8, and 9. Specifically, Fig. 5 shows cases with acoustic shadows being classified as medium-high (a) or very low (b) risk. Figure 6 shows cases without acoustic shadows being classified as medium-high (a) or very low (b) risk. Figure 7 shows cases of solid OMs being classified as medium-high (a) or very low (b) risk. Figure 8 shows cases of cystic OMs being classified as mediumhigh (a) or very low (b) risk. Figure 9 shows cases of mixed OMs being classified as medium-high (a) or very low (b) risk.

\section{Discussion}

AI technology has recently brought an unprecedented growth of applications to medical imaging, and AI predicting models are entering into clinical practice, thanks

Table 2 Second cohort population

\begin{tabular}{llrrrrrr}
\hline & $\begin{array}{l}\text { Number of } \\
\text { patients }\end{array}$ & \multicolumn{2}{c}{$\begin{array}{l}\text { Mass } \\
\text { type }\end{array}$} & \multicolumn{2}{l}{$\begin{array}{l}\text { Median age } \\
\text { (IQR) }\end{array}$} & $\begin{array}{l}\text { Postmenopausal } \\
\text { (\%) }\end{array}$ & \multicolumn{2}{l}{$\begin{array}{l}\text { Malignant } \\
\text { (\%) }\end{array}$} & \multicolumn{2}{l}{$\begin{array}{l}\text { Malignant, median age } \\
\text { (IQR) }\end{array}$} \\
\hline $\begin{array}{l}\text { Second cohort (35 } \\
\text { women) }\end{array}$ & 8 & Solid & $54(9)$ & $6(75.0 \%)$ & $7(87.5 \%)$ & $54(8)$ \\
& & 13 & Liquid & $42(23)$ & $4(30.8 \%)$ & $1(7.7 \%)$ & $68(0)$ \\
& & 14 & Mixed & $54(29)$ & $10(71.4 \%)$ & $12(85.7 \%)$ & $5(23)$ \\
\hline
\end{tabular}


Table 3 Histopathological characteristics of ovarian masses included in the study

\begin{tabular}{|c|c|}
\hline Variable & $\begin{array}{l}\text { Ovarian masses, } \\
\text { number }(\%)\end{array}$ \\
\hline Benign & $132(48.2)$ \\
\hline Malignant & $142(51.8)$ \\
\hline Total & $274(100.0)$ \\
\hline \multicolumn{2}{|l|}{ Histopathological type of benign masses } \\
\hline Serous cystoadenoma/cystoadenofibroma & $48(36.4)$ \\
\hline Mucinous cystoadenoma & $9(6.8)$ \\
\hline Endometrioma & $12(9.1)$ \\
\hline Ovarian fibroma/fibrothecoma & $38(28.8)$ \\
\hline Teratoma & $8(6.1)$ \\
\hline Pelvic inflammatory disease & $2(1.5)$ \\
\hline Peritoneal cyst/pseudocysts & $3(2.3)$ \\
\hline Hydrosalpinx & $7(5.3)$ \\
\hline Paraovarian cyst / paratubaric cyst & $5(3.8)$ \\
\hline Total & $132(100.0)$ \\
\hline \multicolumn{2}{|l|}{ Histopathological type of malignant masses } \\
\hline High-grade serous ovarian cancer & $58(40.9)$ \\
\hline Low-grade serous ovarian cancer & $6(4.2)$ \\
\hline Serous borderline tumour & $12(8.5)$ \\
\hline $\begin{array}{l}\text { Mucinous borderline tumour } \\
\text { gastrointestinal type }\end{array}$ & $7(4.9)$ \\
\hline $\begin{array}{l}\text { Mucinous borderline tumour endocervical } \\
\text { type }\end{array}$ & $5(3.5)$ \\
\hline Mucinous ovarian cancer & $2(1.4)$ \\
\hline Endometrioid ovarian cancer & $4(2.8)$ \\
\hline Clear cell ovarian cancer & $4(2.8)$ \\
\hline Granulosa cell ovarian tumour & $3(2.1)$ \\
\hline Ovarian carcinosarcoma & $5(3.5)$ \\
\hline Dysgerminoma & $1(0.7)$ \\
\hline Ovarian metastases from other tumour & $22(15.5)$ \\
\hline Tubal cancer & $1(0.7)$ \\
\hline Yolk sac tumour & $3(2.1)$ \\
\hline Immature teratoma & $6(4.2)$ \\
\hline Sertoli Leydig tumour & $3(2.1)$ \\
\hline Total & $142(100.0)$ \\
\hline \multicolumn{2}{|l|}{ FIGO stage of primitive ovarian cancers } \\
\hline IA & $26(21.7)$ \\
\hline IB & $2(1.7)$ \\
\hline IC1 & $4(3.3)$ \\
\hline IC2 & $1(0.8)$ \\
\hline IC3 & $6(5.0)$ \\
\hline$\| \mathrm{A}$ & $3(2.5)$ \\
\hline$\| \mathrm{B}$ & $6(5.0)$ \\
\hline$\||| A 1$ & $6(5.0)$ \\
\hline
\end{tabular}

Table 3 Histopathological characteristics of ovarian masses included in the study (Continued)

\begin{tabular}{lr}
\hline Variable & $\begin{array}{c}\text { Ovarian masses, } \\
\text { number (\%) }\end{array}$ \\
\hline IIIA2 & $1(0.8)$ \\
IIIB & $9(7.5)$ \\
IIIC & $37(30.8)$ \\
IVA & $5(4.2)$ \\
IVB & $14(11.7)$ \\
Total & $120(100.0)$ \\
\hline FIGO International Federation of Gynecology and Obstetrics &
\end{tabular}

to the wide availability of digital medical images and the technical advancements in hardware and software architectures compared with the technologies and libraries of the past. AI has been applied to TUS OMs in some studies, albeit not as extensively as some other imaging modalities such as radiography, mammography, computed tomography, and magnetic resonance imaging for solid cancers. An automatic analysis of TUS images based on quantification of gray-level features was proposed by Zimmer et al. [22] obtaining a success rate of $80-90 \%$ for benign OMs but only $70 \%$ for solid and mixed malignant OMs. An expert system [23] and artificial neural networks $[24,25]$ were applied to classify US image into benign and malignant but image features were manually measured and provided by the experimenters. Kazendar et al. [26] developed a fully automatic machine learning classifier stratifying US images as benign or malignant masses with an accuracy of $77 \%$ when images were enhanced with a Local Binary Pattern operator. An automatic scoring system, HistoScanning, based on the quantification of tissue disorganisation induced by malignant processes in backscattered ultrasound waves before image processing was developed as computer-aided technology able to predict malignant versus benign OMs with excellent sensitivity (90\%) and high specificity (88\%). However, this tool should be embedded in the US system to properly function [27].

In our study, we have created, tested, and prospectively validated a predictive model to triage OMs based on radiomic analysis of TUS images combined with menopausal status and CA125 levels. Our model proved to be an accurate and reproducible DSS for the clinicians with an accuracy of $91 \%$ on the validation cohort.

For this purpose, ultrasonography studies from a retrospective cohort of 239 patients and from a prospective cohort of 35 patients were analysed by two different examiners using a stand-alone software tool in which the DSS was implemented, allowing fast manual segmentation of OM, robust radiomic analysis, and classification of risk level of single-subject OMs. 
Table 4 Performances of the decision support system for the two patient cohorts and the two examiners

\begin{tabular}{llll}
\hline & & Examiner $\mathbf{1}$ & Examiner $\mathbf{2}$ \\
\hline First cohort (239 women) & Sensitivity & $122 / 123,99.2 \%(95.6-100.0)$ & $121 / 123,98.4 \%(94.3-99.8)$ \\
& Specificity & $88 / 116,75.9 \%(67.0-83.3)$ & $91 / 116,78.5 \%(69.9-85.5)$ \\
& Accuracy & $210 / 239,87.9 \%(83.0-91.7)$ & $212 / 239,88.7 \%(84.0-92.4)$ \\
& PPV & $122 / 150,81.3 \%(74.2-87.2)$ & $121 / 146,82.9 \%(75.8-88.6)$ \\
Second cohort (35 women) & NPV & $88 / 89,98.9 \%(93.9-100.0)$ & $20 / 20,100.0 \%(83.2-100.0)$ \\
& Sensitivity & $20 / 20,100.0 \%(83.2-100.0)$ & $12 / 15,80.0 \%(51.9-95.7)$ \\
& Specificity & $12 / 15,80.0 \%(51.9-95.7)$ & $32 / 35,91.4 \%(76.9-98.2)$ \\
& Accuracy & $32 / 35,91.4 \%(76.9-98.2)$ & $20 / 23,86.967 \%(66.4-97.2)$ \\
& PPV & $20 / 23,86.967 \%(66.4-97.2)$ & $12 / 12,100.0 \%(74.5-100.0)$ \\
\hline
\end{tabular}

Data are given as ratios, percentages ( $95 \%$ confidence intervals). Note: two of the 35 ovarian masses were defined differently by the two examiners (solid for the first examiner, mixed for the second): however, the decision support system classified them with the same level of risk (medium-high risk)

Compared to our first model developed (AROMA pilot study) [18], we have improved the DSS by introducing clinical and biological parameters easy to be obtained (CA-125 value and menopausal status) and an TUS parameter (presence of shadows) that is simple to identify for the examiners because it is a clear, common, and well-known US image feature associated with benign ovarian masses [28].

We have also tested the reproducibility of the tool with different TUS machines and between examiners with different levels of experience with excellent results. Although the subjective impression of the experienced TUS examiner can perform well in defining those OMs to be surgically treated [29], the lack of reproducibility among different examiners represents one of the highest clinical unmet needs in gynaecologic oncology TUS [30]. Our tool aims to bring this gap providing a fair and reproducible approach for assessing the nature of OMs.

A strength of our study is the radiomic analysis according to the IBSI guidelines of the International Biomarker Standardisation Initiative (IBSI), which is a guarantee of feature extraction reproducibility. Moreover, definitive histological examination after surgery-considered as reference standard-was available for all patients.

Furthermore, the model, classifying patients into two classes (very low risk and medium-high risk) overcomes the problem of the "uncertain" OM class: the current recommendations for the "uncertain" class problem are to assess the OMs by second-level imaging (e.g., magnetic resonance imaging) or to address directly to surgery, with a high number of false positives. With our predictive model, the masses in the very low class can be managed conservatively, while the masses in the medium-high risk class can be assessed by second-level imaging (e.g., magnetic resonance imaging) or surgery, with a reduction of about one third in false positives and false negatives. This dichotomy certainly represents an important decision support for less experienced examiners in OMs triage.

The DSS system showed excellent diagnostic performance, not only for the first cohort of patients (retrospective), used to train and test the DSS, but also for the second one (prospective), used as independent test, both in terms of sensitivity and specificity, demonstrating a high generalisation and reproducibility of results with respect to the two different TUS systems and with respect to the two different examiners. This advantage is warranted by random manipulations of manual segmentation of the OMs by an examiner, simulating variations in segmentations miming different examiner segmentations. Radiomic features are selected as stable among these manipulations and this avoids a certain percentage of intrinsic error.

With respect in particular to the examiner reproducibility, it should be noted that, in the first cohort, all OMs were defined in the same class by the two examiners (solid or cystic or mixed), while in the second cohort two of the 35 OMs were defined differently (solid for the first examiner, mixed for the second one): however, the DSS classified them with the same level of risk (medium-high risk). Considering that all the OMs analysed in this study (both for the retrospective and for the prospective court) were sent to the intervention on the basis of the indications of the medical oncologists' specialists and that the negative predictive value of the DSS is higher than 97\%, the tool showed high potential in avoiding treatments to negative patients while maintaining a high ability in selecting the patients to be referred for surgery.

Of note, the predictive values were quite balanced due to the disease prevalence that was $51.5 \%(123 / 239)$ in the first cohort and $57.1 \%(20 / 35)$ in the second 



Fig. 5 Transvaginal ultrasound of ovarian masses with acoustic shadows: (a) solid mass with acoustic shadows, CA125 205 IU/mL, immature teratoma on histological examination (medium-high risk), (b) mixed adnexal mass with acoustic shadows, benign teratoma on histological examination (very low risk)

independent cohort. This balanced prevalence, as expected [31], enhances the role of the DSS in predicting the risk of malignancy allowing to strongly reduce both false positives and false negatives. We would also specify that all patients enrolled in the study underwent surgery for various reasons: suspicion of malignancy on ultrasound, patient request, symptoms worsening the quality of life, reasons connected with fertility. There were no sources of bias due to the exclusion of very low-risk cases due to human readers.
Our work has some limitations. Compared to the more widely used ADNEX model, our DSS does not stratify the risk of the OM to be a borderline tumour, an early or advanced ovarian cancer or a metastasis $[11,32$, 33]. Moreover, the validation will take advantage of cohorts from more centres and larger sample size. All ultrasound examinations were performed by an experienced reader; thus, images may have been leaning towards a higher-than-average quality. We did not perform a classification of ultrasound images by the two 

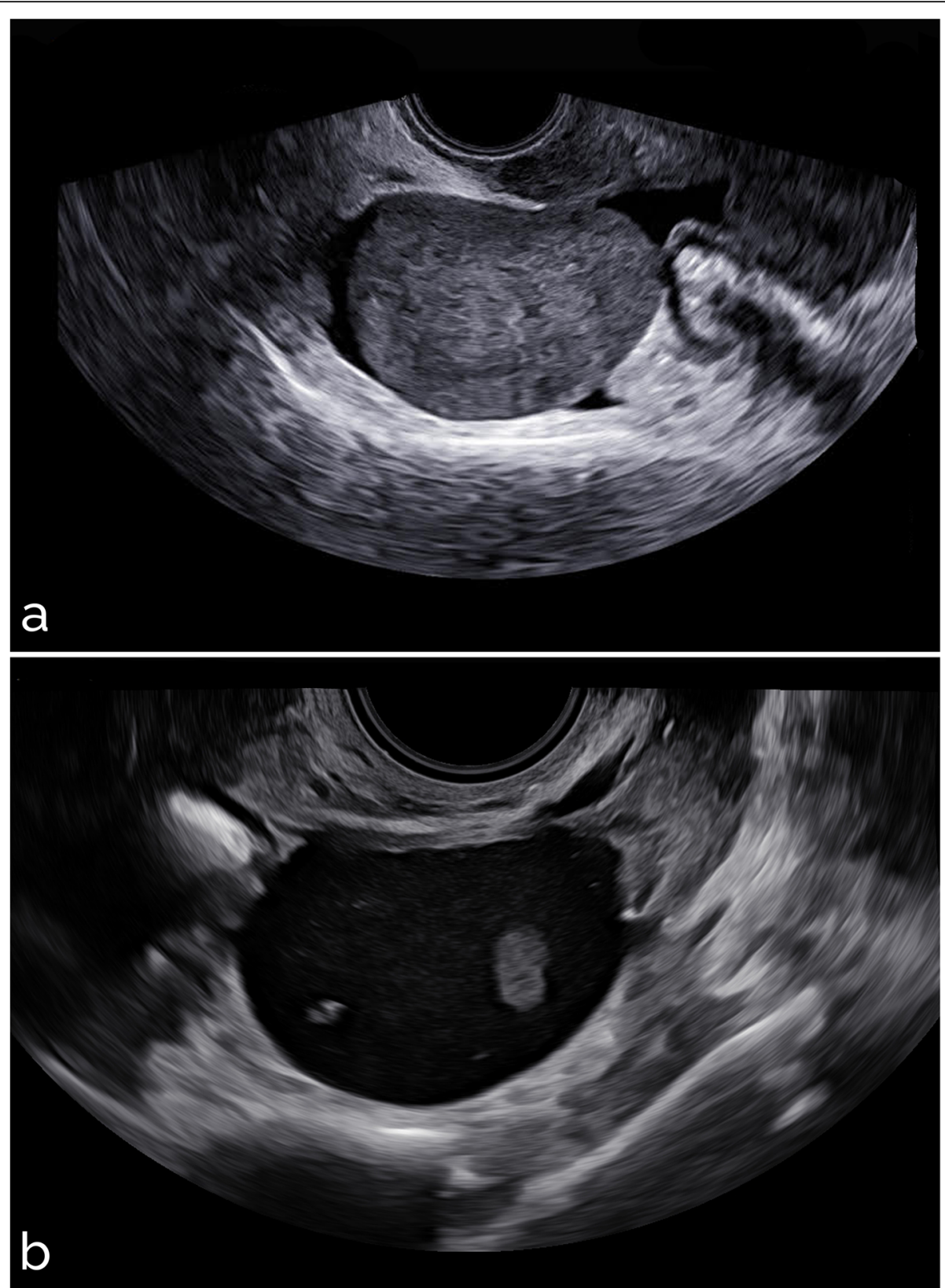

Fig. 6 Transvaginal ultrasound of ovarian masses without acoustic shadows: (a) solid mass without acoustic shadows, CA 125 12 IU/mL, malignant on histological examination (Krukenberg ovarian tumour) (medium-high risk), (b) cystic mass without acoustic shadows, endometrioma at histological examination (very low risk)

human readers into the two risk classes. Given that readers' experience might be a key issue in both ultrasound image acquisition and interpreting findings from ultrasound concerning ovarian cancer, the comparison on the use of the DSS among users of different experiences including the image acquisition step could possibly highlight the fact that less experienced readers, such as those performing ultrasound examinations in centres with limited ovarian cancer workflows, might benefit from our model to a greater extent or, on the contrary, showing a lower performance when ultrasound images are acquired by less skilled operators. More so, it might be interesting to review whether having a human read parallel to the automatic classification system could possibly lead to an even higher accuracy, leaning towards a double-read system combining imaging features and human insight.

In conclusion, our DSS tool for predicting the risk level of malignancy of OMs through combination of TUS features and clinical/biological parameters shows high accuracy. The tool is semi-automated and provides 

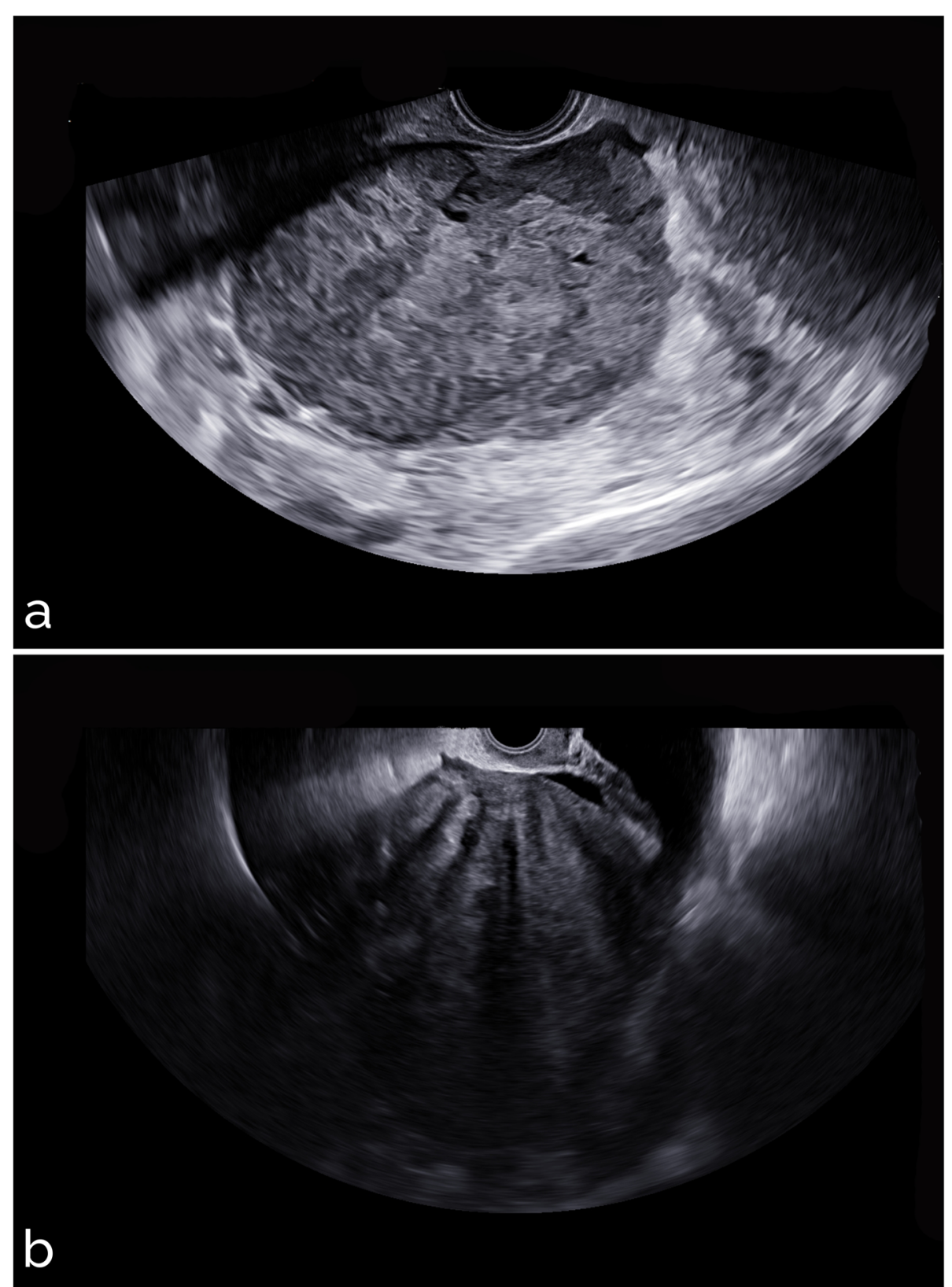

Fig. 7 Transvaginal ultrasound of solid ovarian masses: (a) clear cell ovarian cancer on histological examination (medium-high risk), (b) ovarian fibroma on histological examination (very low risk) 

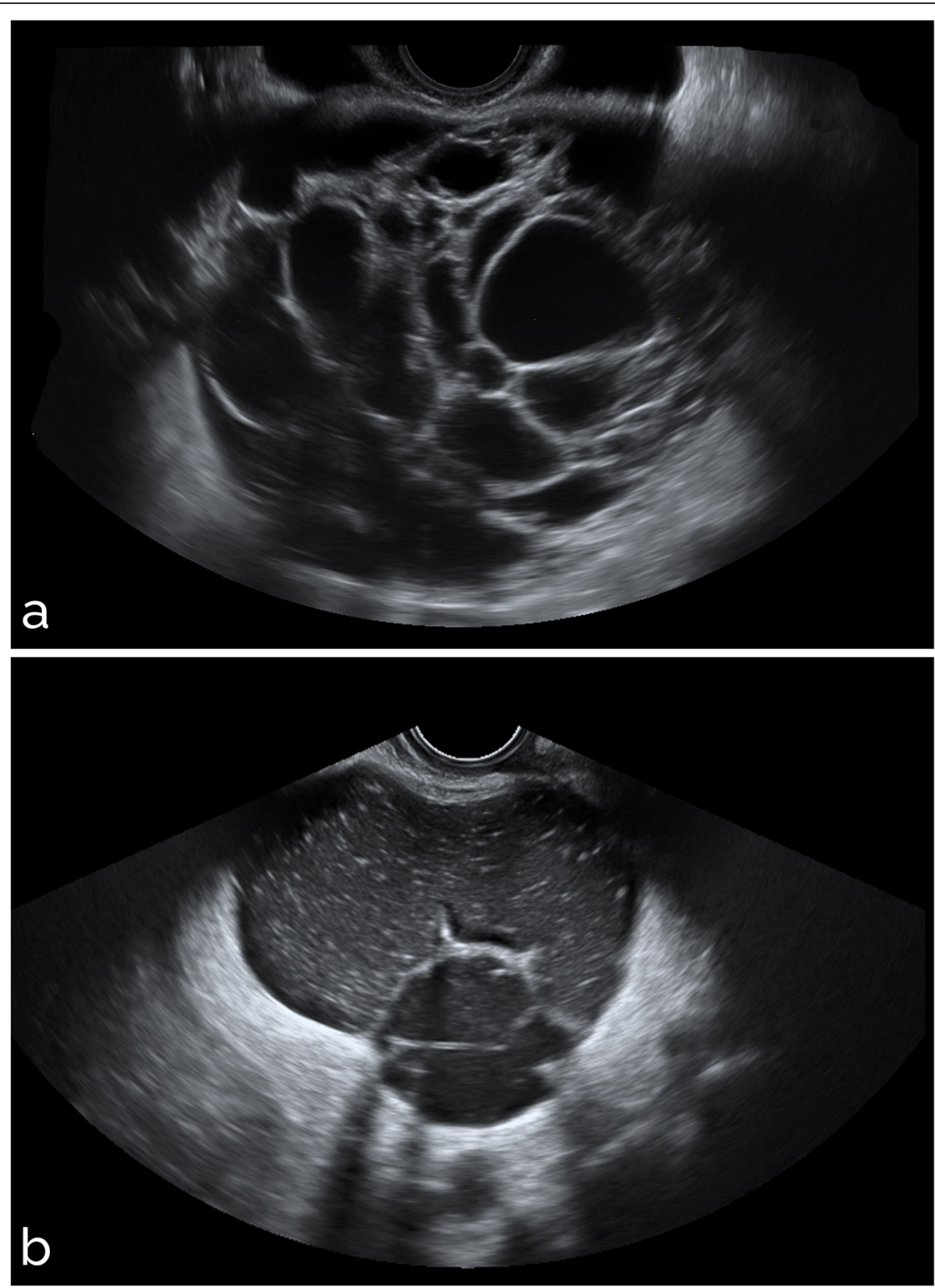

Fig. 8 Transvaginal ultrasound of cystic ovarian masses: (a) mucinous ovarian cancer on histological examination (medium-high risk), (b) mucinous cystoadenoma on histological examination (very low risk) 



Fig. 9 Transvaginal ultrasound of mixed ovarian masses: (a) serous borderline tumour on histological examination (medium-high risk), (b) cystoadenofibroma on histological examination (very low risk)

a reproducible analysis among different TUS machines and examiners that could offer a useful second opinion after an only qualitative visual analysis of TUS images by a single examiner, supporting the clinical decision making. The results obtained in the "real-world" through prospective validation on the considered cohort of patients are exciting and will be monitored on other cohorts in a multicentre setting.

\section{Abbreviations}

ADNEX: Assessment of different neoplasias in the adnexa;" CA-125: Cancer antigen-125; Cl: Confidence interval; DICOM: Digital imaging and communications in medicine; DSS: Decision support system; FIGO: International Federation of Gynecology and Obstetrics; IBSI: International biomarker standardisation initiative; IQR: Interquartile range; OC: Ovarian cancer; OM: Ovarian mass; TUS: Transvaginal ultrasonography

\section{Authors' contributions}

Conceptualisation, VC, GB, IC, FR; methodology and formal analysis, IC, MI, CS; data curation, IC, MI, CS, FB, GS, MS; software CS, writing, VC, GB, MI, CS: review and editing, IC, FR, MS; supervision, IC, FR. All author(S) read and approved the final manuscript.

\section{Funding}

No funding source was obtained. 


\section{Availability of data and materials}

The datasets used and/or analysed during the current study are available from the corresponding author on reasonable request.

\section{Declarations}

\section{Ethics approval and consent to participate}

All procedures performed in studies involving human participants were in accordance with the ethical standards of the institutional and/or national research committee and with the 1964 Declaration of Helsinki and its later amendments or comparable ethical standards.

Patients in the prospective cohort and patients still alive and traceable in the retrospective cohort signed the study-specific informed consent. For deceased or untraceable patients, the Principal Investigator has filled in the "Replacement form for informed consent" approved by the ethics committee, in compliance with DGPR 679/2016

\section{Consent for publication}

Not applicable.

\section{Competing interests}

Isabella Castiglioni and Matteo Interlenghi own DeepTrace Technologies S.R.L shares. Christian Salvatore is the CEO of DeepTrace Technologies S.R.L. DeepTrace Technologies. S.R.L is a spin-off of Scuola Universitaria degli Studi Superiori IUSS, Pavia, Italy. The other authors of this manuscript declare no relationships with any companies, whose products or services may be related to the subject matter of the article.

\section{Author details}

'Department of Gynecologic Oncology, Fondazione IRCCS Istituto Nazionale dei Tumori di Milano, Via Venezian 1, 20133 Milan, Italy. ${ }^{2}$ DeepTrace Technologies S.R.L., Milan, Italy. ${ }^{3}$ Clinical Trial Center, Fondazione IRCCS Istituto Nazionale Tumori di Milano, Milan, Italy. ${ }^{4}$ Dipartimento di Fisica G. Occhialini, University of Milan-Bicocca, Milan, Italy.

Received: 6 May 2021 Accepted: 21 May 2021

Published online: 26 July 2021

\section{References}

1. Ferlay J, Colombet M, Soerjomataram I, et al (2021) Cancer statistics for the year 2020: An overview. Int J Cancer. https://doi.org/10.1002/ijc.33588

2. Ferlay J, Colombet M, Soerjomataram I, et al (2019) Estimating the global cancer incidence and mortality in 2018: GLOBOCAN sources and methods. Int J Cancer 144:1941-1953. https://doi.org/10.1002/ijc.31937

3. Podo F, Sardanelli F, lorio E, et al (2007) Abnormal choline phospholipid metabolism in breast and ovary cancer: molecular bases for noninvasive imaging approaches. Curr Med Imaging Rev 3:123-137. https://doi.org/10.21 74/157340507780619160

4. Menon U, Gentry-Maharaj A, Hallett R, et al (2009) Sensitivity and specificity of multimodal and ultrasound screening for ovarian cancer, and stage distribution of detected cancers: results of the prevalence screen of the UK Collaborative Trial of Ovarian Cancer Screening (UKCTOCS). Lancet Oncol 10:327-340. https://doi.org/10.1016/S1470-2045(09)70026-9

5. Pinsky PF, Yu K, Kramer BS, et al (2016) Extended mortality results for ovarian cancer screening in the PLCO trial with median 15 years follow-up. Gynecol Oncol 143:270-275. https://doi.org/10.1016/j.ygyno.2016.08.334

6. Lowry KP, Lee SI (2017) Imaging and screening of ovarian cancer. Radiol Clin N Am 55:1251-1259. https://doi.org/10.1016/.j.rcl.2017.06.010

7. Woo YL, Kyrgiou M, Bryant A, Everett T, Dickinson HO (2012) Centralisation of services for gynaecological cancers - a Cochrane systematic review. Gynecol Oncol 126:286-290. https://doi.org/10.1016/j.ygyno.2012.04.012

8. Miller RW, Ueland FR (2012) Risk of malignancy in sonographically confirmed ovarian tumors. Clin Obstet Gynecol 55:52-64. https://doi.org/1 0.1097/GRF.0b013e31824970cf

9. Prat J, FIGO Committee on Gynecologic Oncology (2014) Staging classification for cancer of the ovary, fallopian tube, and peritoneum. Int J Gynaecol Obstet 124:1-5. https://doi.org/10.1016/j.ijgo.2013.10.001

10. Jacobs I, Oram D, Fairbanks J, Turner J, Frost C, Grudzinskas JG (1990) A risk of malignancy index incorporating CA 125, ultrasound and menopausal status for the accurate preoperative diagnosis of ovarian cancer. Br J Obstet Gynaecol 97:922-999. https://doi.org/10.1111/j.14710528.1990.tb02448.x

11. Van Calster B, Van Hoorde K, Valentin L et al (2014) Evaluating the risk of ovarian cancer before surgery using the ADNEX model to differentiate between benign, borderline, early and advanced stage invasive, and secondary metastatic tumours: prospective multicentre diagnostic study. BMJ 349:g5920. https://doi.org/10.1136/bmj.g5920

12. Timmerman D, Valentin L, Bourne TH, et al (2000) Terms, definitions and measurements to describe the sonographic features of adnexal tumors: a consensus opinion from the International Ovarian Tumor Analysis (IOTA) Group. Ultrasound Obstet Gynecol 16:500-505. https://doi.org/10.1046/j.14 69-0705.2000.00287.x

13. Nougaret S, Tardieu M, Vargas HA, et al (2019) Ovarian cancer: an update on imaging in the era of radiomics. Diagn Interv Imaging 100:647-655. https://doi.org/10.1016/j.diii.2018.11.007

14. Kumbhare D, Shaw S, Ahmed S, Noseworthy MD (2020) Quantitative ultrasound of trapezius muscle involvement in myofascial pain: comparison of clinical and healthy population using texture analysis. J Ultrasound 23: 23-30. https://doi.org/10.1007/s40477-018-0330-5

15. Chiappa V, Interlenghi M, Salvatore C, et al (2021) Using rADioMlcs and machine learning with ultrasonography for the differential diagnosis of myometRiAL tumors (the ADMIRAL pilot study). Radiomics and differential diagnosis of myometrial tumors. Gynecol Oncol 161:838-844. https://doi. org/10.1016/j.ygyno.2021.04.004

16. Yeh AC, Li H, Zhu Y, et al (2019) Radiogenomics of breast cancer using dynamic contrast enhanced MRI and gene expression profiling. Cancer Imaging 19:48. https://doi.org/10.1186/s40644-019-0233-5

17. Aerts HJ, Velazquez ER, Leijenaar RT et al (2014) Decoding tumour phenotype by noninvasive imaging using a quantitative radiomics approach. Nat Commun 5:4006 https://doi.org/10.1038/ncomms5006. Erratum in: Nat Commun. 2014;5:4644. Cavalho, Sara [corrected to Carvalho, Sara]

18. Chiappa V, Bogani G, Interlenghi M, et al (2020) The Adoption of Radiomics and machine learning improves the diagnostic processes of women with Ovarian MAsses (the AROMA pilot study). J Ultrasound https://doi.org/10.1 007/s40477-020-00503-5

19. Karlsen MA, Sandhu N, Høgdall C, et al (2012) Evaluation of HE4, CA125, risk of ovarian malignancy algorithm (ROMA) and risk of malignancy index (RMI) as diagnostic tools of epithelial ovarian cancer in patients with a pelvic mass. Gynecol Oncol 127:379-383. https://doi.org/10.1016/j.ygyno.2012.07.106

20. ACOG Committee on Gynecologic Practice (2003) The role of the generalist obstetrician-gynecologist in the early detection of ovarian cancer. Int J Gynaecol Obstet 80:235-238. https://doi.org/10.1016/s0020-7292(03)00022-5

21. ACOG Committee on Practice Bulletins-Gynecology (2016) Practice Bulletin No. 174: Evaluation and management of adnexal masses. Obstet Gynecol 128:e210-e226. https://doi.org/10.1097/AOG.0000000000001768

22. Zimmer $Y$, Tepper R, Akselrod S (2003) An automatic approach for morphological analysis and malignancy evaluation of ovarian masses using B-scans. Ultrasound Med Biol 29:1561-1570. https://doi.org/10.1016/j.ultra smedbio.2003.08.013

23. Bruning J, Becker R, Entezami M et al (1997) Knowledge-based system ADNEXPERT to assist the sonographic diagnosis of adnexal tumors. Methods Inf Med 36:201-206. https://doi.org/10.1055/s-0038-1636830

24. Tailor A, Jurkovic D, Bourne TH, Collins WP, Campbell S (1999) Sonographic prediction of malignancy in adnexal masses using an artificial neural network. Br J Obstet Gynaecol 106:21-30. https://doi.org/10.1111/j.1471-052 8.1999.tb08080.x

25. Biagiotti R, Desii C, Vanzi E, Gacci G (1999) Predicting ovarian malignancy: application of artificial neural networks to transvaginal and color Doppler flow US. Radiology 210:399-403. https://doi.org/10.1148/radiology.210.2. regfe 18399

26. Khazendar S, Sayasneh A, Al-Assam H et al (2015) Automated characterisation of ultrasound images of ovarian tumours: the diagnostic accuracy of a support vector machine and image processing with a local binary pattern operator. Facts Views Vis Obgyn 7:7-15

27. Lucidarme O, Akakpo JP, Granberg S et al (2010) A new computer-aided diagnostic tool for non-invasive characterisation of malignant ovarian masses: results of a multicentre validation study. Eur Radiol 20:1822-1830. https://doi.org/10.1007/s00330-010-1750-6

28. Sladkevicius P, Valentin L (2014) Interobserver agreement in describing the ultrasound appearance of adnexal masses and in calculating the risk of 
malignancy using logistic regression models. Clin Cancer Res 21:594-601. https://doi.org/10.1158/1078-0432.CCR-14-0906

29. Timmerman D (2004) The use of mathematical models to evaluate pelvic masses; can they beat an expert operator? Best Pract Res Clin Obstet Gynaecol 18:91-104. https://doi.org/10.1016/j.bpobgyn.2003.09.009

30. Coelho Neto MA, Roncato P, Nastri CO, Martins WP (2015) True

Reproducibility of UltraSound Techniques (TRUST): systematic review of reliability studies in obstetrics and gynecology. Ultrasound Obstet Gynecol 46:14-20. https://doi.org/10.1002/uog.14654

31. Zhang Z, Bullock RG, Fritsche H (2019) Adnexal mass risk assessment: a multivariate index assay for malignancy risk stratification. Future Oncol 15: 3783-3795. https://doi.org/10.2217/fon-2019-0479

32. Van Calster B, Van Hoorde K, Froyman W et al (2015) Practical guidance for applying the ADNEX model from the IOTA group to discriminate between different subtypes of adnexal tumors. Facts Views Vis Obgyn 7:32-41

33. Van Calster B (2017) External validation of ADNEX model for diagnosing ovarian cancer: evaluating performance of differentiation between tumor subgroups. Ultrasound Obstet Gynecol 50:406-407. https://doi.org/10.1002/ uog. 17391

\section{Publisher's Note}

Springer Nature remains neutral with regard to jurisdictional claims in published maps and institutional affiliations.

\section{Submit your manuscript to a SpringerOpen ${ }^{\circ}$ journal and benefit from:}

- Convenient online submission

- Rigorous peer review

- Open access: articles freely available online

High visibility within the field

- Retaining the copyright to your article

Submit your next manuscript at $\boldsymbol{\nabla}$ springeropen.com 